\title{
MULTIPLE ARTERY ANEURYSMS FOUND DURING ACUTE CORONARY SYNDROME INVESTIGATION. AN UNREPORTED ASSOCIATION
}

Ricardo Ribeiro Dias, Marcelo Franken, Ernesto Lopez, José Carlos Nicolau, Charles Mady, Noedir Antonio Groppo Stolf

\section{INTRODUCTION}

Since the first report of coronary artery aneurysms, ${ }^{1}$ several investigators have reported multiple aneurysm associations from many different arteries. The rare association of coronary aneurysm with intracranial aneurysm is reported. ${ }^{2}$ More often associations between coronary and abdominal aortic aneurysm has also been described. ${ }^{3,4}$ This paper reports a patient with an association of artery aneurysms that has never been reported before.

\section{CASE REPORT}

A 64-year-old man with previously known coronary artery disease presented with acute coronary syndrome in the last two hours. Physical examination showed tachycardia (180beats/min), blood pressure was $100 \times 60 \mathrm{mmHg}$, and ECG showed ventricular tachycardia. Successful electrical cardioversion was performed and sinus rhythm with nonspecific changes in ventricular repolarization was observed. Chest X-ray revealed aorta enlargement and serum troponin levels were undetected. Coronary angiography was performed and showed occluded left circumflex artery and a $90 \%$ obstruction in the left anterior descending coronary artery followed by an aneurysm (fig. 1A). On CT angiogram, a $10.1 \mathrm{~cm}$ descending thoracic aortic aneurysm (fig. 1B), a $4.0 \mathrm{~cm}$ abdominal aortic aneurysm and a $6.8 \mathrm{~cm}$ right iliac artery aneurysm (fig. 1C) were observed. Cerebral angioresonance showed a $1.8 \mathrm{~cm}$ basilar artery aneurysm (fig. 1D).

Two surgeries were programmed during the same hospitalization. The first one consisted of ligation of the LAD coronary aneurysm, coronary bypass with LIMA to LAD, saphenous vein graft to left circumflex artery, followed by

Cardiovascular Surgery Heart Institute - University of São Paulo Medical School - São Paulo/SP, Brazil

Email: diasrr@hotmail.com
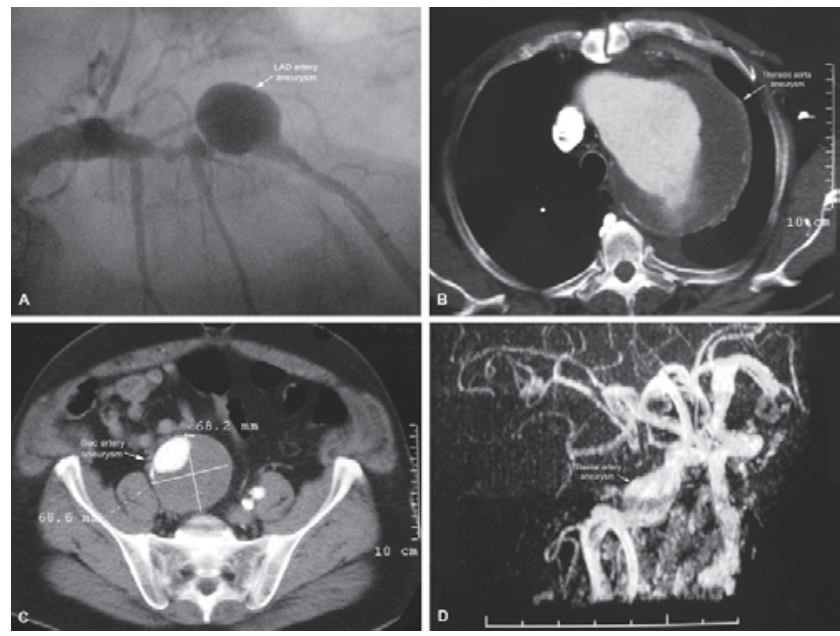

Figure 1 - A - Coronary angiography of left anterior descending coronary artery aneurysm. B - CT angiogram of descending thoracic aorta aneurysm. C - CT angiogram of right iliac artery aneurysm. D - Cerebral angioresonance of basilar artery aneurysm.

exclusion of the descending thoracic aneurysm with an endovascular aortic stent through the aortic arch approach in total circulatory arrest. After recovery, an aortic bi-iliac graft was performed, because the patient had a $4.0 \mathrm{~cm}$ infra renal aortic aneurysm and a $6.8 \mathrm{~cm}$ right iliac artery aneurysm. Pathology findings showed that all lesions were atherosclerotic. All the procedures were uneventful and the patient was discharged with neurological and cardiovascular follow up.

\section{DISCUSSION}

As aneurysms in general, the coronary artery aneurysm is defined as a coronary dilatation that exceeds 1.5 times the diameter of normal adjacent segments or the diameter of the patient's largest coronary vessel ${ }^{5}$.

Based upon antemorten angiographic studies, the incidence of coronary aneurysms varies from $1.5 \%$ to $4.9 \%$ in the presence of coronary artery disease. ${ }^{4}$ In spite of a close 
correlation between abdominal aortic aneurysm and coronary artery disease, the association with coronary aneurysm is less frequent and often related to atherosclerotic disease ${ }^{3}$ as it is in this report. Other rare manifestations of coronary aneurysm associated with multiple peripheral vascular aneurysms reported were due to hyperhomocysteinemia. ${ }^{6}$ The association of coronary and intracranial aneurysms is uncommon, being restricted to few cases. ${ }^{2}$

The surgical intervention of coronary artery aneurysm ligation is performed to eliminate potential complications of expansion, rupture and embolization. Treatment of the descending aorta with an aortic stent through the arch is a combined procedure that allows surgical fixation of the stent in an ideal landing zone

An extensive review of literature revealed no reports of associated intracranial artery aneurysm, coronary artery aneurysm, descending and abdominal aortic aneurysms, and iliac artery aneurysm. Therefore, this case is reported on account of its rarity.

\section{REFERENCES}

1. Scott DH. Aneurysm of coronary arteries. Am Heart J 1948;36:403-21.

2. Harikrishnan S, Stigimon J, Tharakan JM. Intracranial aneurysms, coronary aneurysms and descending aortic coartation - unreported association. Int J Cardiol 2005;99:329-30.

3. LaMendola CL, Culliford AT, Harris LJ, Amendo MT. Multiple aneurysms of coronary arteries in a patient with systemic aneurismal disease. Ann Thorac Surg 1990;49:1009-10.
4. Peker O, Özisik K, Islamoglu F, Posacioglu H, Demircan M. Multiple coronary artery aneurysms combined with abdominal aortic aneurysm. Jpn Heart J 2001;42:135-141.

5. Robertson T, Fisher L. Prognostic significance of coronary artery aneurysm and ectasia in the Coronary Artery Surgery Study (CASS) registry. Prog Clin Biol Res 1987;250:325-9.

6. Wong A, Naik M, Chan C, Chua YL, Chua T. Giant coronary aneurysms with multiple vascular aneurysms: a rare manifestation of hyperhomocysteinemia. Cathet Cardiovasc Intervent 2001;52:116-9. 